\title{
Analysis of Research Hotspots on Big Data in Education: From Bibliometrics Perspective
}

\author{
Weiwei Wang \\ School of Public Administration, South China Normal University, Guangzhou 510006, China \\ wangww@m.scnu.edu.cn
}

\begin{abstract}
This paper used data retrieved from Web of Science database as datasets to analyze the research area of big data in education. Based on the bibliometrics method, the basic characteristics of authors, institutions, and cited references, et al. are described. The visualization of keywords co-occurrence map was presented using Cite Space software. Based on the discussion above, this paper summarized the research status, hotspots and trends.
\end{abstract}

Keywords: big data; education; learning analysis; co-occurrence analysis.

\section{教育大数据研究热点探析: 文献计量学的视角}

\author{
王巍巍 \\ 华南师范大学, 广东广州 中国
}

摘 要: 本研究以WoS数据库中教育大数据的相关研究为分析对象, 采用文献计量的方法, 对 研究文献及研究者的基本特征进行描述, 利用CiteSpace软件对文献关键词共现进行可视化分 析, 在此基础上, 对教育大数据研究的现状、热点及趋势进行了探讨。

关键词：教育大数据; 学习分析; 数据挖掘; 共现分析

\section{1. 前言}

2012 年开始, 大数据一词开始引起广泛关注。大数据被认为是 21 世纪的黄金和石油, 并 将开启重大的时代转型 ${ }^{[1]}$ 。各国纷纷加入大数据研究的行列。2012 年, 联合国发布大数据白 皮书 “Big Data for Development: Challenges \& Opportunities” [2]，2012 年 3 月，美国启动 “Big Data Research and Development Initiative” 计划 ${ }^{[3]}$ 。随后, 包括教育领域在内的多个研究领域 都兴起了对大数据时代各个领域的研究。美国教育部 2012 年 10 月发布 “Enhancing Teaching and Learning through Educational Data Mining and Learning Analytics” 报告 ${ }^{[4]}$, 推动大数据在教 育领域的应用。

为了了解教育大数据领域目前的研究状况和研究热点, 本文采取文献计量学的方法, 通 过对近年来已发表的有关教育大数据的论文进行文献计量分析, 利用 Cite Space 软件对研究 领域热点进行探析, 了解教育大数据领域的发展现状及趋势。

\section{2. 研究方案}

\section{1. 数据来源与数据预处理}

研究数据集的获取主要以Web of Science (Wos) 核心数据集 (包括SCIE、SSCI和A\&HCI) 为检索来源数据库。由于 WoS涵盖期刊范围较全, 且引文格式规范, 是引文分析常用的来源 数据库。

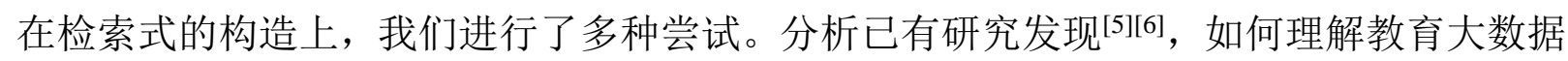
这一概念对检索结果影响很大。在已有研究中, 有的将 “data analytics” “data mining” 等都 独立作为获取教育大数据研究文献的检索词, 但是数据挖掘和数据分析是教育领域已有的研 
究领域, 这样检索出的结果不能准确体现大数据背景下教育应用的发展, 会将检索结果扩大。 通过多次检索尝试和对检索结果进行文献分析, 本文确定将检索式定为"big data" in 主题 AND education* OR “learn* analytics” OR teach* in 主题，检索时间范围是 2004-2016（根据初步检索 结果分析, 符合检索式的文献 2004 年以后才出现, 因此此处将时间范围限定为 2004 年以后。），检索 时间为 2016 年 6 月 11 日，检索结果为 253 条。这样的限定是一方面是为了排除数据集中有 关大数据算法的研究, 如 “machine learning” 等, 同时在一定程度上约束有关教育数据分析 等研究是在大数据背景下探讨的。

\section{2. 工具选择}

对于数据集的分析主要采用 WoS 自带的检索结果分析工具和陈超美博士开发的文献信息 可视化工具 Cite Space 软件。WoS 自带的检索结果分析工具主要用于文献信息基本特征描述, Cite Space 软件用于基于共现分析的研究热点及趋势探析, 是目前比较常用的文献信息可视化 工具。

\section{3. 研究领域基本状态分析}

研究领域基本状态分析主要是对检索结果集中文献数量及作者等基本特征进行统计描 述，包括每年发文数量、作者发文数量、机构发文数等，从整体了解研究领域发展的概貌。

3.1 论文数量及类型分布

从每年的发文数量来看, 如图 1 所示, 教育大数据的研究论文从 2012 年开始迅速成倍增 长, 由于检索时间为 2016 年，所以目前 2016 年的统计数据尚不完整，但是从发展趋势线来 看，未来教育大数据领域的论文还将持续增长。

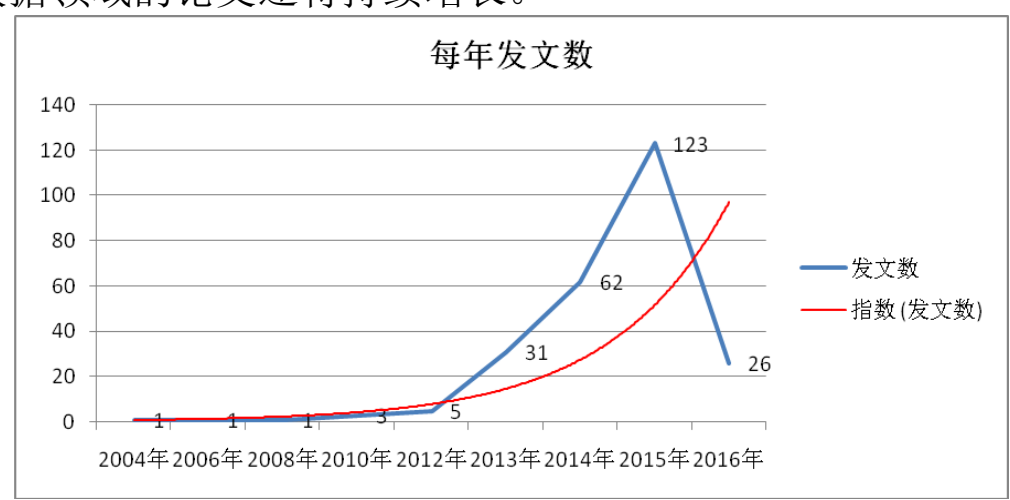

图1 每年发文数

从文献类型来看，所发表的文献中， $45 \%$ 为期刊论文， $44 \%$ 为会议论文，这两种类型的 文献占所有文献数量的近九成, 其它为研究评论、图书章节等。在信息技术及其相关研究领 域, 很多研究成果会选择以会议论文的形式发表, 因此教育大数据的研究论文近半数是会议 论文，这与其以信息技术为支撑的本质是密切相关的。其中发表教育大数据论文最多的期刊 包括 “ADVANCES IN SOCIAL SCIENCE EDUCATION AND HUMANITIES RESEARCH” 发 文 17 篇，“PROCEDIA COMPUTER SCIENCE” 发文 8 篇，会议 “JOINT INTERNATIONAL SOCIAL SCIENCE EDUCATION LANGUAGE MANAGEMENT AND BUSINESS CONFERENCE JISEM” 发文 4 篇。在所有发表的文献中，受美国国家科学基金资助的论文数 有 12 篇，是资助基金中发表论文最多的，中国国家自然科学基金资助论文数有 2 篇。

从国家/地区的发文数量来看，美国发文最多达到 96 篇，这与近年来美中两国在大数据 研究领域投入的政策鼓励和资金投入不无关系。紧随其后的分别为中（55 篇）、英（18 篇）、 澳大利亚（13 篇）和加拿大（12 篇）。

\section{2 作者及机构信息}

表1所示为作者发文数量排行，排名前两位的作者XU YJ和ZHANG JH的所属机构均为北 京师范大学通识教育中心珠海校区, 且两位是多篇教育大数据论文的合著者。作者XU YJ发 表的五篇论文中四篇发布于JISEM2015（JOINT INTERNATIONAL SOCIAL SCIENCE EDUCATI 
ON LANGUAGE MANAGEMENT AND BUSINESS CONFERENCE）会议，另外一篇是发布于201 4年的会议论文, 可以推测在该机构中有一个探索教育大数据研究的科研合作团队。

发文量同为3篇的作者WALLER MA和FAWCETT SE也是合著者。从两位作者发表的论文 主题来看, 以大数据在供应链管理中的应用为主, 兼论及课程教育问题, 属于低相关研究。 另外两位发文量同为3篇的作者MODELSKI J和ROMANIUK R同样为 3 篇论文的合著者。他们 发表的论文是有关电子和电信技术在波兰的应用探讨, 发表于 2010 年的会议上, 其研究论及 目前热门的大数据技术以及技术对教育的影响，也属于低相关研究。

表1 作者发文数量

\begin{tabular}{cccc}
\hline 序号 & 作者 & 发文数 & 所属机构 \\
\hline 1 & XU YJ & 5 & 北京师范大学通识教育中心珠海校区 \\
\hline 2 & ZHANG JH & 3 & 北京师范大学通识教育中心珠海校区 \\
\hline 3 & WALLER MA & 3 & 美国阿肯色大学 \\
\hline 4 & FAWCETT SE & 3 & 美国韦伯州立大学 \\
\hline 5 & MODELSKI J & 3 & 波兰华沙理工大学 \\
\hline 6 & ROMANIUK R & 3 & 波兰华沙理工大学 \\
\hline
\end{tabular}

从机构发文数量来看, 发文最多的为加州大学系统, 共有 9 篇论文, 其次为北京师范大学 (7篇) 和斯坦福大学 (6篇) 等, 其中发文量排名前十位的除了北京师范大学以外均为美国 的大学, 由此可见美国的大学在教育大数据研究中走得较快, 是目前的主要研究力量。

3.3 文献被引情况

文献被引用次数越多表明该文献在所属研究领域中的权威性越高, 基础性地位越重, 同 时也能够在一定程度上反映出所属研究领域的研究重点。表 2 为被引次数排名前十位的文献, 从题名中可以看出，前九位均为低相关文献，第十位为高度相关文献。

表2 高被引文献

\begin{tabular}{|c|c|c|c|}
\hline 序号 & 文献题名 & $\begin{array}{c}\text { 出版 } \\
\text { 年 }\end{array}$ & $\begin{array}{c}\text { 被引次 } \\
\text { 数 }\end{array}$ \\
\hline 1 & Business Intelligence and Analytics: From Big Data to Big Impact & 2012 & 190 \\
\hline 2 & $\begin{array}{c}\text { Data Science, Predictive Analytics, and Big Data: A Revolution That Will Transform } \\
\text { Supply Chain Design and Management }\end{array}$ & 2013 & 33 \\
\hline 3 & $\begin{array}{c}\text { Testing regimes, accountabilities and education policy: commensurate global and } \\
\text { national developments }\end{array}$ & 2013 & 21 \\
\hline 4 & Making Advanced Analytics Work For You & 2012 & 19 \\
\hline 5 & $\begin{array}{c}\text { Electronics and telecommunications in Poland, issues and perspectives Part III: } \\
\text { Innovativeness, Applications, Economy, Development Scenarios, Politics }\end{array}$ & 2010 & 17 \\
\hline 6 & $\begin{array}{c}\text { Electronics and telecommunications in Poland, issues and perspectives Part II: } \\
\text { Science, Research, Development, Higher Education }\end{array}$ & 2010 & 14 \\
\hline 7 & $\begin{array}{c}\text { How Can Research Keep Up With eHealth? Ten Strategies for Increasing the } \\
\text { Timeliness and Usefulness of eHealth Research }\end{array}$ & 2014 & 13 \\
\hline 8 & $\begin{array}{c}\text { Electronics and telecommunications in Poland, issues and perspectives Part I: Society } \\
\text { and Education }\end{array}$ & 2010 & 13 \\
\hline 9 & Developing the role of big data and analytics in health professional education & 2014 & 12 \\
\hline 10 & Data entry: towards the critical study of digital data and education & 2015 & 11 \\
\hline
\end{tabular}

对第十位文献进行文献分析，找到作者 Selwyn N 来自澳大利亚莫纳什大学，该文献 2015 年 1 月发表于 “LEARNING MEDIA AND TECHNOLOGY” 期刊, 是由斯宾塞基金会 (Spencer Foundation) 资助。在该文献中作者概述了教育领域数字数据的重要性, 对数据不公平、数据 在组织和控制管理模式中的角色、数字监控 (dataveillance) 等问题做了探讨, 并给出了未来 数字数据和教育的研究框架。

上述分析可以看出，被引频率较高的文献均为探讨大数据及其在商业、健康等领域的应 用研究, 尽管排名前十位被引频率较高的文献中只有最后一位是教育大数据领域高相关研究, 
但是该研究2015年发表以来就已经达到11次的引用量, 引用年增长率较高, 属于WoS中的高 引用率文献, 可见教育大数据的关注度较高。

\section{4. 基于共现分析的研究热点及发展趋势探析}

共现分析的原理是将数据集中的文献关键词共现频率，确定关键词之间的关联关系。一 般来讲, 共现频率越高, 说明两个关键词之间的关系越密切。通过关键词共现分析可以发现 一个研究领域中关系密切的研究主题, 并可以根据关键词中心度的大小（即与其它关键词共 现的频率大小），确定核心研究领域。

\section{1 研究热点分析}

使用Cite Space软件对数据集中各文献的关键词进行共现分析，截取出出现频次最高的关 键词及其中心度如表3左半部所示。其中出现频次最多的为 “big data”、“education” 和

“technology”，表明这一领域内。大数据、教育与技术是最受关注的主题。除此外，教育领 域特有的关键词如 “learning analytics”、“higher education”、“curriculum” 和 “students” 出现的频次也较高, 体现出我们研究的数据集指向教育领域的特征。

表3 关键词出现频次和中心度

\begin{tabular}{|c|c|c|c|c|c|c|c|}
\hline 序号 & 关键词 & 频次 & 中心度 & 序号 & 关键词 & 中心度 & 频次 \\
\hline 1 & big data & 107 & 0.35 & 1 & big data & 0.35 & 107 \\
\hline 2 & education & 32 & 0.22 & 2 & education & 0.22 & 32 \\
\hline 3 & technology & 16 & 0.17 & 3 & technology & 0.17 & 16 \\
\hline 4 & learning analytics & 13 & 0.08 & 4 & analytics & 0.17 & 7 \\
\hline 5 & cloud computing & 9 & 0.08 & 5 & higher education & 0.13 & 8 \\
\hline 6 & higher education & 8 & 0.13 & 6 & biology & 0.11 & 5 \\
\hline 7 & social media & 8 & 0.1 & 7 & social media & 0.1 & 8 \\
\hline 8 & impact & 7 & 0.02 & 8 & service & 0.09 & 3 \\
\hline 9 & networks & 7 & 0.08 & 9 & learning analytics & 0.08 & 13 \\
\hline 10 & analytics & 7 & 0.17 & 10 & cloud computing & 0.08 & 9 \\
\hline
\end{tabular}

表3中的右半部分为按照中心度进行的关键词排名，与左半部对比发现，两个排名中的关 键词相差不多, 其中灰色部分的两个关键词是左半部分没有的, 这两个关键词中心度较高, 表明它们跟其它关键词共同出现的频率较高, 可以推测部分研究领域是与教育大数据服务有 关的，还有在生物领域的大数据或教育大数据研究。

利用CiteSpace软件进行关键词的共现分析, 获得图2所示的关键词共现网络, 该网络包含 204个节点, 214条链接。图中所显示出来的短语为设定共现频率大于 4 时的关键词, 此时的网 络基本可以反映出教育大数据研究领域涵盖的主要主题。图中短语的字体越大来表该短语的 共现次数越高, 可以看出共现次数最多的为“big data”和“education”, 还有 “learning analytics”、 “higher education”、“cloud computing”、“curriculum” 等，是该研究领域的核心关键词， 该图也是表3中所示的高中心度关键词及其关系的直观显示。此外图2中各关键词之间的链接 表示关键词之间的共现关系, 链接的粗细表明共现关系的强弱, 可以看出 “data analysis” 和 “privacy” 两个关键词虽然中心度不高, 但是他们之间的链接强度较大, 可以推测有关数据 分析的研究很多会和隐私保护问题的探讨联系在一起。 


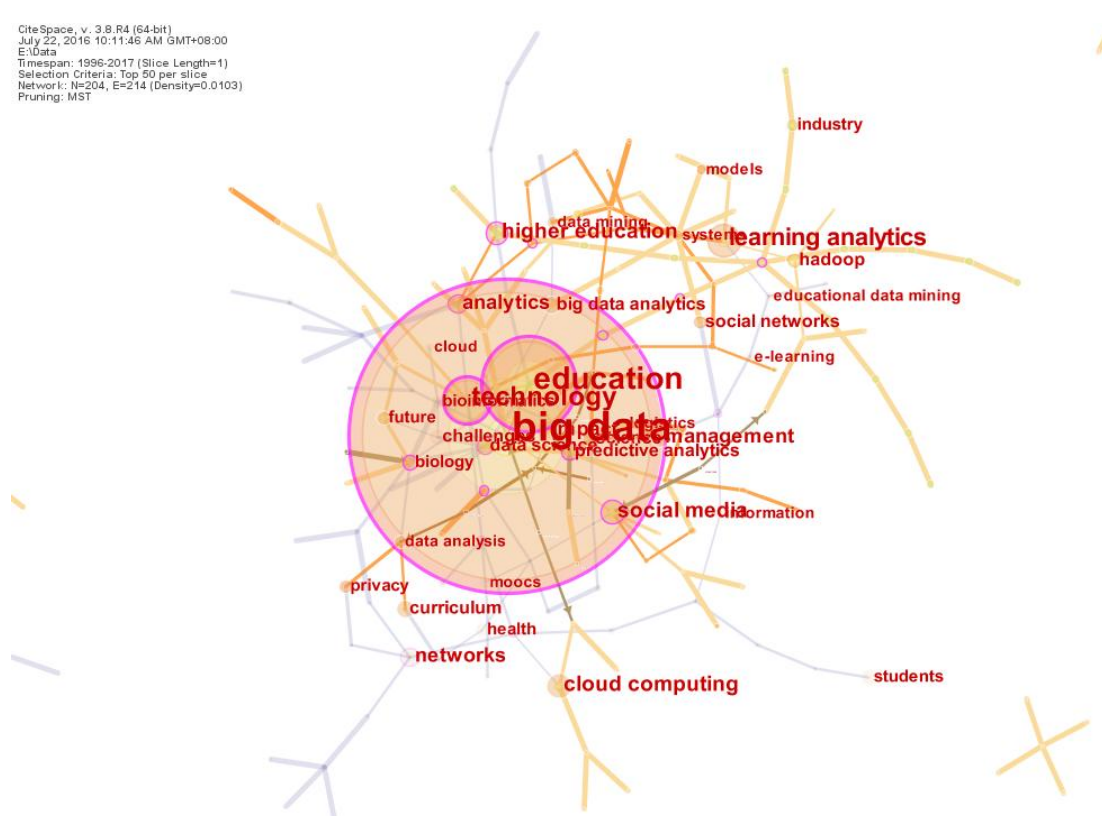

\section{2 研究趋势分析}

\section{图2 关键词共现网络}

在研究领域发展过程中出现的突现词被认为更能体现出某一阶段研究的新动向和新变

化, 因此CiteSpace软件的突现词检测 (Burst Terms) 功能经常被用来判断研究的发展发展方 向和趋势。我们利用CiteSpace软件获得关键词突现词, 整理后获得7个相关度较高的突现词, 如表4所示。从表中可以看出2014年开始 “e-learning” 和 “education” 的研究突然增多, 反映 出这段时间有关教育大数据及e-learning开始引起广泛关注, 是教育大数据研究开始大规模发 展的阶段。

表4 关键词的突现词及其强度

\begin{tabular}{|c|c|c|c|c|}
\hline 序号 & 突现词 & 突现强度 & 开始时间 & 结束时间 \\
\hline 1 & Electronics and telecommunications & 5.2688 & 2009 & 2011 \\
\hline 2 & e-learning & 4.6288 & 2014 & 2014 \\
\hline 3 & education & 4.6288 & 2014 & 2014 \\
\hline 4 & Predictive analytics & 4.2604 & 2013 & 2013 \\
\hline 5 & Information technology & 3.7863 & 2013 & 2013 \\
\hline 6 & Open source & 3.2414 & 2014 & 2014 \\
\hline 7 & Engineering education & 3.2383 & 2014 & 2014 \\
\hline
\end{tabular}

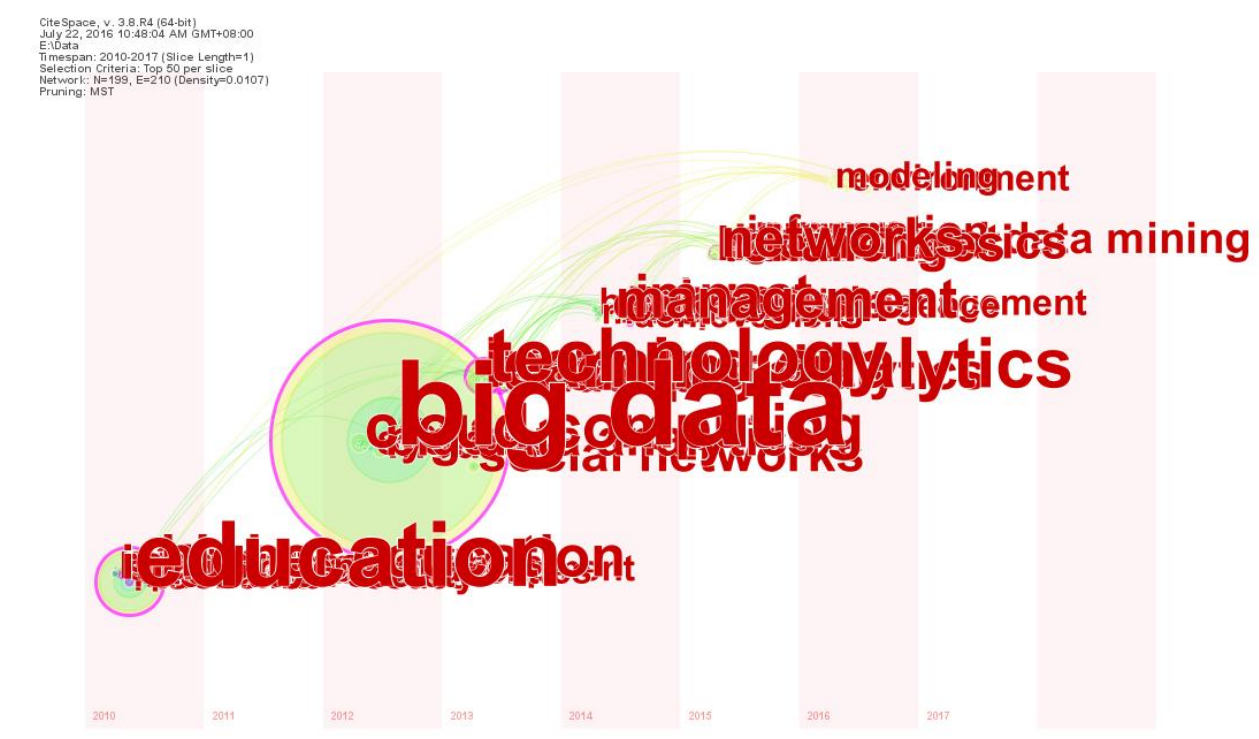

图3 关键词共现的时序分析 
对关键词共现网络进行时序图绘制, 看以看出研究热点的变迁及发展趋势。为了使图形 易看, 我们将数据集中2004年的四篇孤岛文献删除, 绘制出下面的图像, 如图3所示。可以看 出2012年为大数据研究热点出现的一年, 探讨大数据的研究剧增。2014年开始较多出现 “data mining”、“data analysis”、“mooc” 等与教育直接相关的关键词, 2015年 “e-learning”、 “students”等关键词增多，到了2016年，开始出现 “modeling”、“smart education”、

“visualization” 等关键词。由此可见, 尽管大数据研究是从2012年开始兴起, 同年联合国、 美国等也发布了推进教育大数据研究的政策计划, 但是知道2014年以后, 教育大数据的研究 才开始进入快速发展期。

\section{5. 结论与讨论}

通过对教育大数据研究领域文献的计量学特征描述, 以及采用 CiteSpace 软件对文献进行 了关键词共现分析, 得出以下结论:

(1) 教育大数据的发展可以划分为三个阶段: 2012 年以前是发展的准备期, 这一阶段开 始关注利用信息技术解决教育领域的问题; 2012 年-2013 年为萌芽期, 大数据研究开始蓬勃 发展, 但是教育大数据领域的研究尚未形成规模; 2014 年-2016 年为发展期, 这一阶段大数 据与教育领域真正开始碰撞出火花, 各类教育大数据研究开始迅速增多, 关注的研究问题也 不断拓展, 从技术到隐私, 再到课程设计和可视化, 研究不断涌现。

（2）教育大数据的研究热点至少包含以下三个方面: 一是教育大数据技术研究; 二是教 育数据挖掘和学习分析研究; 三是教育大数据的应用, 包括课程设计、学生学习和可视化研 究等。

（3）教育大数据研究的发展趋势: 从研究内容来看, 未来的教育大数据研究将更多关注 用户需求, 如用户建模、学生学习需求等, 还有教育大数据带来的诸多管理、隐私保护、数 据监控等问题; 从研究主体来看, 目前大数据研究的主力为美国各高校, 未来将会有更多国 家更多类型的研究机构加入其中。

\section{致谢}

感谢教育部人文社会科学规划青年基金项目（项目编号：16JYC880078）、广东省教育厅青 年创新人才项目（项目编号：2015WQNCX015）的资助。

\section{参考文献}

[1] Mayer-schonberger V, Cukier K. Big Data. Zhejiang People's publishing House, 2013, p. 09.

[2] Information on: http://www.unglobalpulse.org/projects/BigDataforDevelopment

[3] Information on: https://www.whitehouse.gov/sites/default/files/microsites/ostp/big_data_press _release_final_2.pdf

[4] Information on: http://tech.ed.gov/wp-content/uploads/2014/03/edm-la-brief.pdf

[5] Wang J, Chen S, Wang L, Yang X. The Analysis of Research Hot Spot and Trend on Big Data in Education based on CiteSpace. Modern Education Technology, Vol.26 (2016) No.02, p.5-13.

[6] He X, Huang L. Visualization Analysis of Evolution Path, Research Hotspots and Frontiers of Big Data. Journal of Modern Information. Vol.35 (2015)No.4,p:46-51 